\title{
Optical and magnetooptical properties of bismuth and gallium substituted iron garnet films
}

\author{
Fredrik Hansteen ${ }^{\mathrm{a}, \mathrm{b}, *}$, Lars Egil Helseth ${ }^{\mathrm{c}}$, Tom H. Johansen ${ }^{\mathrm{d}}$, Ola Hunderi ${ }^{\mathrm{a}}$, Andrei Kirilyuk ${ }^{\mathrm{b}}$, \\ Theo Rasing ${ }^{\mathrm{b}}$ \\ ${ }^{a}$ The Norwegian University of Science and Technology, 7491 Trondheim, Norway \\ ${ }^{\mathrm{b}}$ NSRIM University of Nijmegen, Toernooiveld 1, 6525 ED Nijmegen, The Netherlands \\ ${ }^{\mathrm{c} M a x}$ Planck Institute of Colloids and Interfaces, D-14424 Potsdam, Germany \\ ${ }^{\mathrm{d}}$ Department of Physics, University of Oslo, P.O. Box 1048, Blindern, Norway
}

\begin{abstract}
A series of iron garnet films of composition $\mathrm{Lu}_{3-x} \mathrm{Bi}_{x} \mathrm{Fe}_{5-y} \mathrm{Ga}_{y} \mathrm{O}_{12}$ grown on (100) oriented GGG substrates have been studied using variable angle spectroscopic ellipsometry and polar Kerr spectroscopy (MOKE). The diagonal and off-diagonal components of the permittivity tensor have been determined in the range 1-5 eV. Our results seem to be in accordance with previously reported measurements on similar systems. We find that the optical absorption is reduced as the gallium content is increased. With increased $\mathrm{Ga}$ doping we also see an increase in the real part of $\varepsilon_{0}$ above $3.7 \mathrm{eV}$, but a decrease for lower energies. In the spectra of the off-diagonal permittivity tensor components we observe bismuth enhancement and gallium dilution effects.
\end{abstract}

(C) 2003 Elsevier B.V. All rights reserved.

Keywords: Ellipsometry; Optical properties; Garnets; Magnetooptics

\section{Introduction}

Garnets, since their discovery in the 1950s, have been extensively studied, driven by the prospects of technological applications [1,2]. Magnetic storage media (bubble memories), sensors, displays, modulators and switches are some of the numerous applications that have been suggested. Despite the efforts, not all aspects of these materials are fully understood. In particular, the transitions responsible for their optical and strong magnetooptical response are still a matter of debate $[3,4]$. A detailed theoretical understanding and accurate experimental results are needed in order to gain further insight into the microscopic physics of these materials.

Previous experimental studies of the optical spectra of garnets have mainly been based on reflectivity measurements and Kramers-Kronig analysis to obtain the diagonal components of the permittivity tensor $[5,6]$. Ellipsometry is a more reliable and very sensitive technique, which can accurately measure the optical properties of materials, however, it also relies on careful

\footnotetext{
*Corresponding author. Tel.: +31-24-3653-093; fax: +31-243652-190.

E-mail address: fredrik.hansteen@phys.ntnu.no (F. Hansteen).
}

modeling of the system under study and fitting of experimental data in order to obtain optical constants.

We have studied a series of $2.5-4-\mu \mathrm{m}$ thick garnet films of composition

$\mathrm{Lu}_{3-x} \mathrm{Bi}_{x} \mathrm{Fe}_{5-y} \mathrm{Ga}_{y} \mathrm{O}_{12}$

using variable angle spectroscopic ellipsometry and spectroscopic MOKE. We have determined for three selected samples the diagonal and off-diagonal permittivity tensor components in the energy range $1-5 \mathrm{eV}$.

The permittivity tensor is commonly used as the link between experimental results and microscopic theory of materials. The linear optical response of our garnet films can be described by a permittivity tensor of the form

$\varepsilon=\left(\begin{array}{lll}\varepsilon_{0} & i \varepsilon_{1} & 0 \\ -i \varepsilon_{1} & \varepsilon_{0} & 0 \\ 0 & 0 & \varepsilon_{0}\end{array}\right)$

when the magnetization is in the $z$-direction (polar configuration). For an absorbing medium the tensor components are complex and given by $\varepsilon_{\mathrm{j}}=\varepsilon^{\prime}{ }_{\mathrm{j}}+\varepsilon^{\prime \prime}{ }_{\mathrm{j}}$. To 
the first order in the magnetization the diagonal component $\varepsilon_{0}$ is independent of $\vec{M}$. The off-diagonal component $\varepsilon_{1}$ is linear in $\vec{M}$ and gives rise to magnetooptical effects. At normal incidence the Kerr rotation $\theta_{\mathrm{K}}$ and ellipticity $\eta_{\mathrm{K}}$ of a semi-infinite medium are related to the components of the permittivity tensor through the relation [7]

$\Phi_{\mathrm{K}}=\theta_{\mathrm{K}}+i \eta_{\mathrm{K}}=\frac{-i \varepsilon_{1}}{\sqrt{\varepsilon_{0}}\left(\varepsilon_{0}-1\right)}$

For more complex systems such as thin films or multilayer structures, no simple relation exists, and numerical fitting of measurements to model calculations should be employed in order to determine off-diagonal tensor elements from Kerr measurements and knowledge of $\varepsilon_{0}$.

\section{Experimental procedure}

The series of garnet films under study have in-plane magnetization and were grown by LPE on (100) oriented gadolinium gallium garnet (GGG) substrates for the purpose of magnetooptical imaging. They are transparent in the near infrared and visible spectral region up to approximately $2.3 \mathrm{eV}$.

Using a commercial ellipsometer system from Woollam Co., Inc., the samples were measured at three angles of incidence, 67,70 and $73^{\circ}$, at photon energies ranging from 1 to $5 \mathrm{eV}$. The measured ellipsometric quantities $\Psi$ and $\Delta$ were then fitted to a model consisting of a GGG substrate, a film layer of unknown thickness and unknown optical constants, and a surface roughness layer (effective medium layer consisting of a $50 \%$ mixture of the previous layer and empty space). The garnet film thicknesses had previously been measured using SEM, and these values were taken as the starting point for the fitting procedure. Below approximately 2.3 $\mathrm{eV}$ the absorption of the garnet films drops and they become transparent, leading to strong interference oscillations in the measurements. In this low energy range of the spectrum, a Cauchy dispersion model was used to represent the optical constants of the film. After obtaining a good fit of the film thickness, surface roughness layer thickness and the parameters of the Cauchy model in this spectral region, the thickness of the garnet film and the surface roughness layer were kept fixed, and $\varepsilon^{\prime}{ }_{0}$ and $\varepsilon^{\prime \prime}{ }_{0}$ fit for every measurement point. Some results of the fitting procedure are summarized in Table 1.

Kerr rotation and ellipticity were also measured using the ellipsometer at $15^{\circ}$ incidence. The samples were mounted on top of a permanent magnet giving a field of $250 \mathrm{kA} / \mathrm{m}$ at the film surface, enough to saturate their magnetization in the out-of-plane direction. Meas-
Table 1

Composition and properties of the garnet samples; film thickness $d_{\mathrm{SEM}}$ determined by SEM, film thickness $d_{\text {elli }}$ and surface roughness layer thicknesses $d_{\text {rough }}$ found by fitting ellipsometry data

\begin{tabular}{llll}
\hline Composition & $d_{\text {SEM }}(\mu \mathrm{m})$ & $d_{\text {elli }}(\mathrm{nm})$ & $d_{\text {rough }}(\mathrm{nm})$ \\
\hline $\mathrm{Lu}_{2.5} \mathrm{Bi}_{0.5} \mathrm{Fe}_{5} \mathrm{O}_{12}$ & 3.0 & 2798 & 6.6 \\
$\mathrm{Lu}_{2.3} \mathrm{Bi}_{0.7} \mathrm{Fe}_{4.7} \mathrm{Ga}_{0.3} \mathrm{O}_{12}$ & 3.5 & 3460 & 4.6 \\
$\mathrm{Lu}_{2.3} \mathrm{Bi}_{0.7} \mathrm{Fe}_{4.4} \mathrm{Ga}_{0.6} \mathrm{O}_{12}$ & 4.0 & 3915 & 7.3 \\
\hline
\end{tabular}

urements were made with the magnetic field in both directions, and the average results used in order to eliminate any offset. The acquired data was again fit to a model to obtain the off-diagonal tensor components. The model was essentially the same as previously described, only modified to include the magnetooptical response of the film.

In addition to the ellipsometric Kerr measurements a home-built photoelastic modulator based setup, similar to that described by Sato [8], was used to verify the results. The sensitivity of this system is greater than that of the ellipsometer, but for our films the ellipsometer proved sufficient. In the absorbing region of the spectrum Eq. (2) can be used to directly estimate the offdiagonal elements, neglecting the small angle of incidence and surface roughness effects.

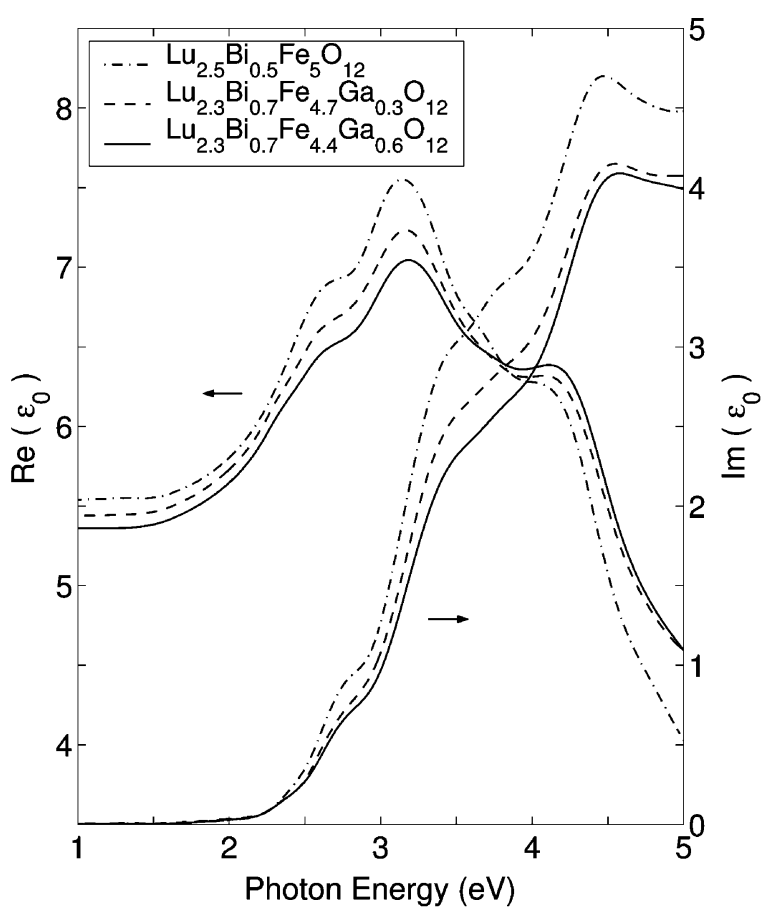

Fig. 1. Diagonal components $\varepsilon_{0}$ of the permittivity tensor for three garnet films of different composition. Gallium substitution significantly reduces the optical absorption. The real part of $\varepsilon_{0}$ shows a decrease at low energies but an increase above $3.7 \mathrm{eV}$ for the samples with higher $\mathrm{Bi}$ and $\mathrm{Ga}$ concentrations. 


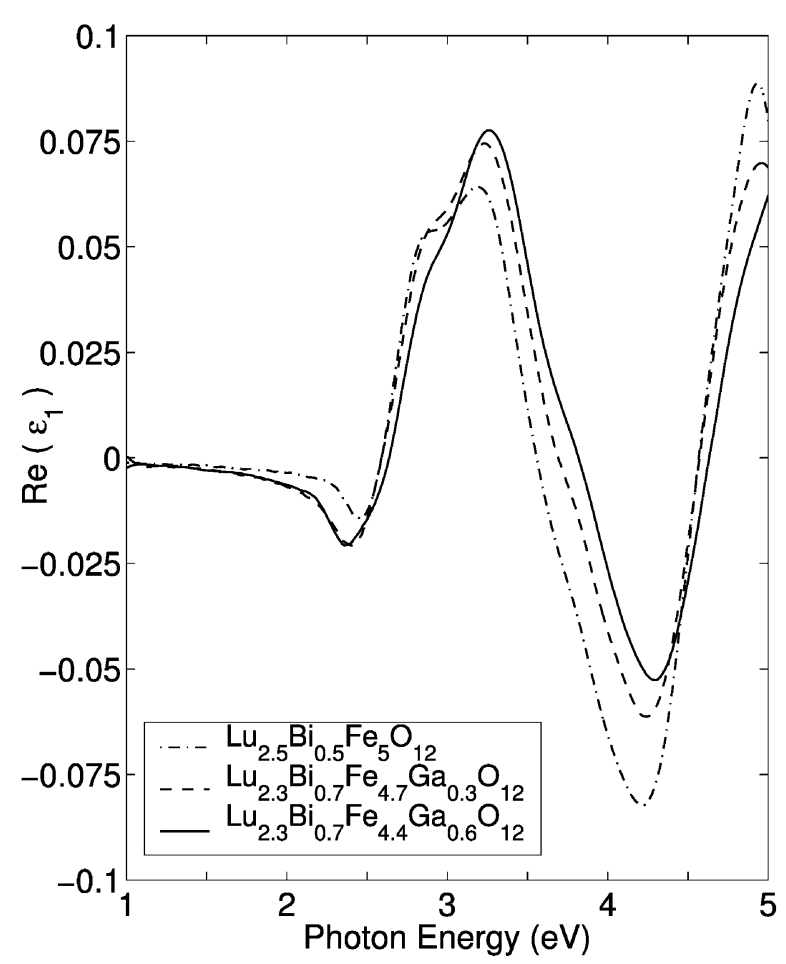

Fig. 2. Real part of the off-diagonal tensor component $\varepsilon_{1}$. Enhancement due to bismuth substitution can be seen below $2.5 \mathrm{eV}$ and for the peak at $3.2 \mathrm{eV}$. Dilution effects of gallium are observed at the 4.2 eV peak.

\section{Results and discussion}

Bismuth substitution is known to increase the refractive index of garnets in the low energy region of the spectrum and to increase the absorption [6,9]. Gallium, however, substitutes the active iron ions at tetrahedral and octahedral sites, and is expected to reduce optical absorption as well as magnetooptical effects. $\mathrm{Ga}^{3+}$ substitutes preferentially for tetrahedrally coordinated $\mathrm{Fe}^{3+}$ (approx. 90\%) and permits thereby in principle for discrimination between the spectral contribution of octahedral and tetrahedral $\mathrm{Fe}^{3+}[5,10]$.

The real and imaginary parts of the diagonal permittivity tensor components for our three samples are shown in Fig. 1. Absorption, essentially due to $\varepsilon^{\prime \prime}{ }_{0}$, can be seen to decrease noticeably for the two samples with higher bismuth $(x=0.7)$ and gallium $(y=0.3, y=0.6)$ concentration. Furthermore, increased gallium substitution seems to reduce $\varepsilon_{0}^{\prime}$ in the low energy part of the spectrum but leading to an increase above $3.7 \mathrm{eV}$. These observations must mainly be attributed to the increase in gallium concentration, and indicate that the effect of diluting the $\mathrm{Fe}^{3+}$ lattice is greater than the effect of added bismuth.

Bismuth substitution of garnets is well known to enhance magnetooptical effects, while Gallium is known to reduce the effects for reasons just mentioned. The

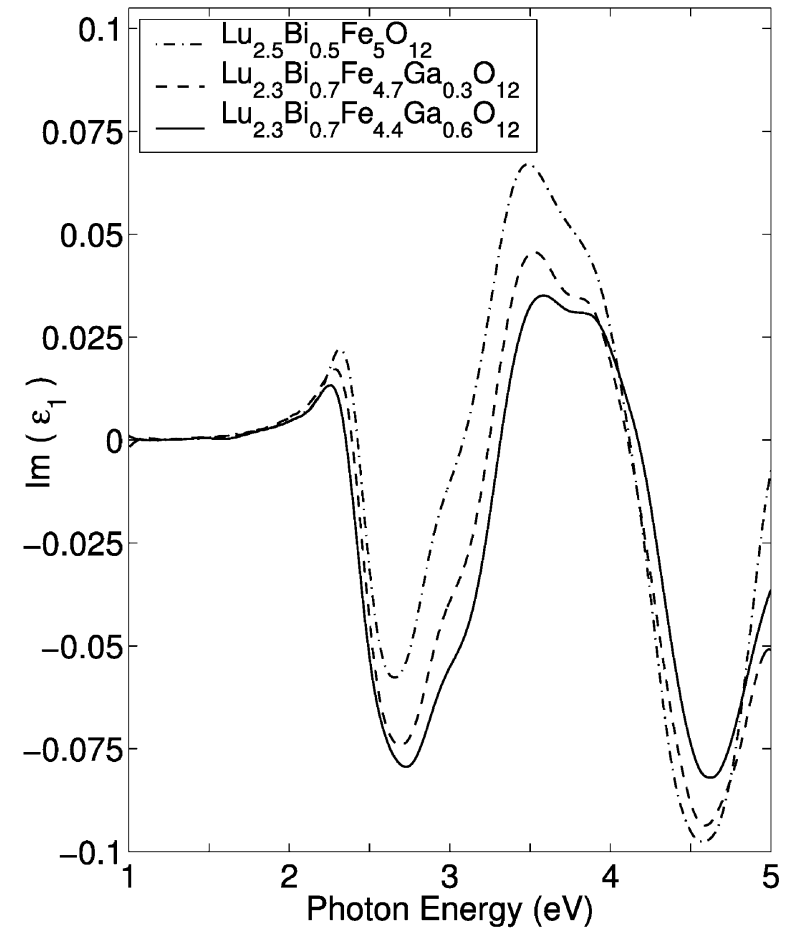

Fig. 3. Imaginary part of the off-diagonal tensor component $\varepsilon_{1}$. Enhancement due to bismuth substitution can be seen for the peak at $2.7 \mathrm{eV}$. Gallium dilution effects are observed for the peaks at 2.3, 3.5 and $4.6 \mathrm{eV}$.

real and imaginary parts of $\varepsilon_{1}$ found from our measurements are shown in Figs. 2 and 3, respectively. Some peaks clearly show the expected dilutive effect of gallium, such as the peaks in $\varepsilon^{\prime \prime}{ }_{1}$ at $2.3,3.5$ and $4.6 \mathrm{eV}$ and in $\varepsilon^{\prime}{ }_{1}$ at $4.2 \mathrm{eV}$. Enhancement due to increased bismuth concentration can be observed in the low energy region of $\varepsilon^{\prime}$, for the peak at $3.2 \mathrm{eV}$, and for the peak in $\varepsilon^{\prime \prime}{ }_{1}$ at $2.7 \mathrm{eV}$. Here gallium dilution seems to have no effect, or in fact the opposite of the expected effect. There also appears to be a pronounced red-shift of the $2.4 \mathrm{eV}$ peak in $\varepsilon^{\prime}{ }_{1}$ and a blue-shift at $4.2 \mathrm{eV}$ for $y=0.3$ and $y=0.6$. Fitting $\varepsilon^{\prime}{ }_{1}$ and $\varepsilon^{\prime \prime}{ }_{1}$ in terms of electricdipole transitions, like several authors have attempted [4,6,11-13], might provide a better understanding of these spectra and the effect of $\mathrm{Bi}$ and $\mathrm{Ga}$ on the transitions involved. Such a fitting should, however, be based on reliable knowledge of the positions and character of the relevant transitions. Without this information the fitting is not unique, and does not further contribute to the understanding of these materials.

The model used to invert the ellipsometry data assumes that the garnet film is homogeneous and that $\varepsilon_{0}$ does not vary through the film. This assumption may not hold completely true, as indicated by magnetization induced second harmonic generation (MSHG) studies of the same samples. This technique, that has a particular sensitivity to symmetry breaking surfaces and interfaces 
[14], shows a difference in crystal symmetry for the signal originating from the film surface and interface. To what extent this has an influence on the linear optical and magnetooptical properties is not known.

\section{Conclusions}

We have experimentally determined the spectra of the permittivity tensor components $\varepsilon_{0}$ and $\varepsilon_{1}$ in the range $1-5 \mathrm{eV}$ for three lutetium iron garnet films doped with gallium and bismuth. There is clearly an influence of the film composition on both $\varepsilon_{0}$ and $\varepsilon_{1}$, however, not always simply to enhance (in the case of bismuth) or reduce (in the case of gallium) spectral features througout the whole spectrum. Theoretical fitting of these spectra is necessary to further understand the results and the microscopic physics of these materials.

\section{References}

[1] G. Winkler, Magnetic Garnets, Friedr. Vieweg and Sohn, 1981.

[2] A. Paoletti, Physics of Magnetic Garnets, Enrico Fermi International School of Physics, Italian Physical Society, NorthHolland Publishing Co, 1978.

[3] A. Zenkov, A. Moskvin, Bismuth-induced increase of the magneto-optical effects in iron garnets: a theoretical analysis, J. Phys.: Condens. Matter 14 (2002) 6957-6968.

[4] G.A. Allen, G.F. Dionne, Accurate analysis of the magnetooptical permittivity tensor of $\mathrm{Y}_{3} \mathrm{Fe}_{5} \mathrm{O}_{12}$, J. Appl. Phys. 93 (10) (2003) 6951-6953.
[5] F.J. Kahn, P. Pershan, J. Remeika, Ultraviolet magneto-optical properties of single-crystal orthoferrites, garnets, and other ferric oxide compounds, Phys. Rev. 186 (3) (1969) 891-918.

[6] S. Wittekoek, T.J.A. Popma, J.M. Robertson, P.F. Bongers, Magneto-optic spectra and the dielectric tensor elements of bismuth-substituted iron garnets at photon energies between 2.2-5.2 eV, Phys. Rev. B 12 (7) (1975) 2777-2788.

[7] A. Zvezdin, V. Kotov, Modern Magnetooptics and Magnetooptical Materials, IOP Publishing Ltd, 1997.

[8] K. Sato, Measurement of magneto-optical Kerr effect using piezo-birefringent modulator, J. Appl. Phys. 20 (12) (1981) 2403-2409.

[9] P. Hansen, C.-P. Klages, J. Schuldt, K. Witter, Magnetic and magneto-optical properties of bismuth-substituted lutetium iron garnet films, Phys. Rev. B 31 (9) (1985) 5858-5864.

[10] G. Scott, D. Lacklison, J. Page, The effects of octahedral $\mathrm{Fe}^{3+}$ and tetrahedral $\mathrm{Fe}^{3+}$ dilution on the Faraday spectra of bismuth-doped iron garnets, J. Phys. C 8 (1975) 519-529.

[11] Z. Šimša, H. Le Gall, J. Šimšová, J. Koláček, A.L. Le PaillierMalécot, Spectral dependences of Faraday rotation in $\mathrm{Y}_{3-x} \mathrm{Bi}_{x} \mathrm{Fe}_{5} \mathrm{O}_{12}$ LPE films, IEEE Trans. Magn. 20 (5) (1984) 1001-1003.

[12] G.F. Dionne, G.A. Allen, Spectral origins of giant Faraday rotation and ellipticity in Bi-substituted magnetic garnets, J. Appl. Phys. 73 (10) (1993) 6127-6129.

[13] L. Helseth, R. Hansen, E. Il'yashenko, M. Baziljevich, T. Johansen, Faraday rotation spectra of bismuth-substituted ferrite garnet films with in-plane magnetization, Phys. Rev. B 64 (2001) 174406.

[14] K.H. Bennemann, Nonlinear optics in metals, International Series of Monographs on Physics, Oxford Clarendon Press, 1998, Ch. 3. 